Supplementary Material for

\title{
Intermetallic Communication through Carbon Wires in Heterobinuclear Cationic Allenylidene Complexes of Chromium
}

Normen Szesni, ${ }^{\mathrm{a}}$ Matthias Drexler, ${ }^{\mathrm{a}}$ Jörg Maurer, ${ }^{\mathrm{b}}$ Rainer F. Winter, ${ }^{\mathrm{b}}$ Frédéric de Montigny, ${ }^{\mathrm{c}}$ Claude Lapinte, ${ }^{\mathrm{c}}$ Stefan Steffens, ${ }^{\mathrm{d}}$ Jürgen Heck, ${ }^{\mathrm{d}}$ Bernhard Weibert, ${ }^{\mathrm{a}}$ and Helmut Fischer ${ }^{\mathrm{a} *}$

${ }^{a}$ Fakultät für Chemie, Universität Konstanz, Postfach 5560 M727, D-78434 Konstanz, Germany

${ }^{b}$ Institut für Anorganische Chemie, Universität Regensburg, Universitätsstraße 31, D93040 Regensburg, Germany

${ }^{c}$ UMR CNRS 6226 Sciences Chimiques de Rennes, Université de Rennes I, Campus Beaulieu, 35042 Rennes Cedex, France

${ }^{d}$ Institut für Anorganische und Angewandte Chemie, Universität Hamburg, MartinLuther-King-Platz 6, D-20146 Hamburg, Germany 


\section{Complex 2a:}

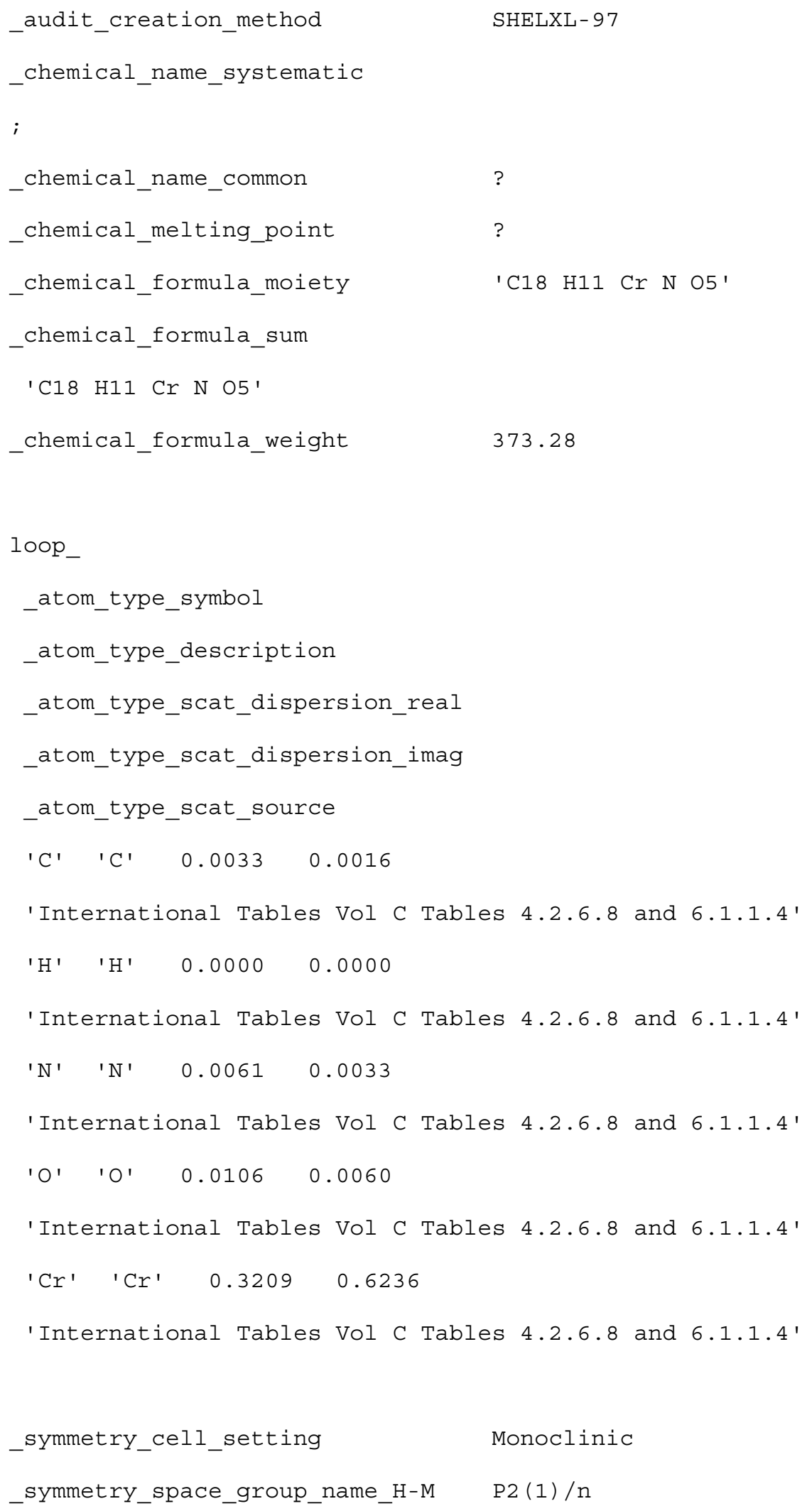




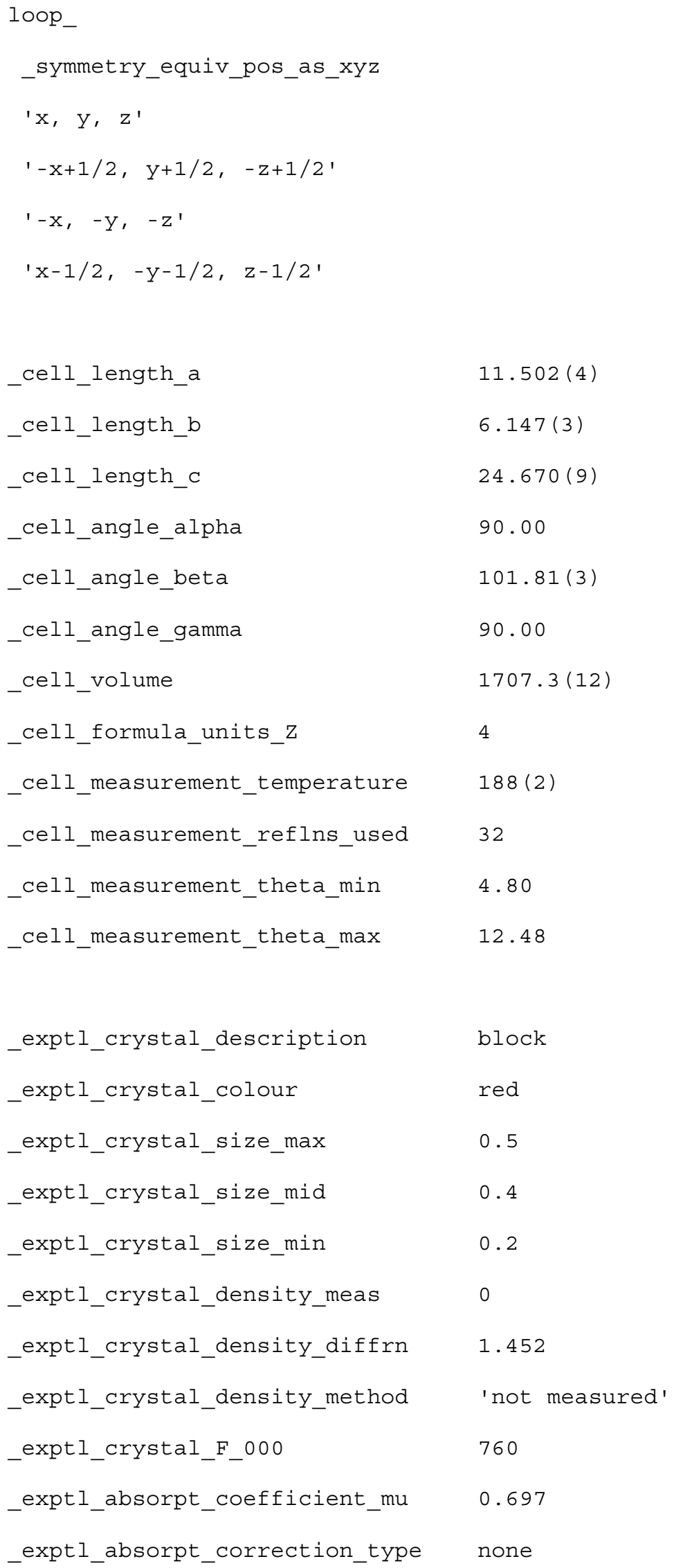




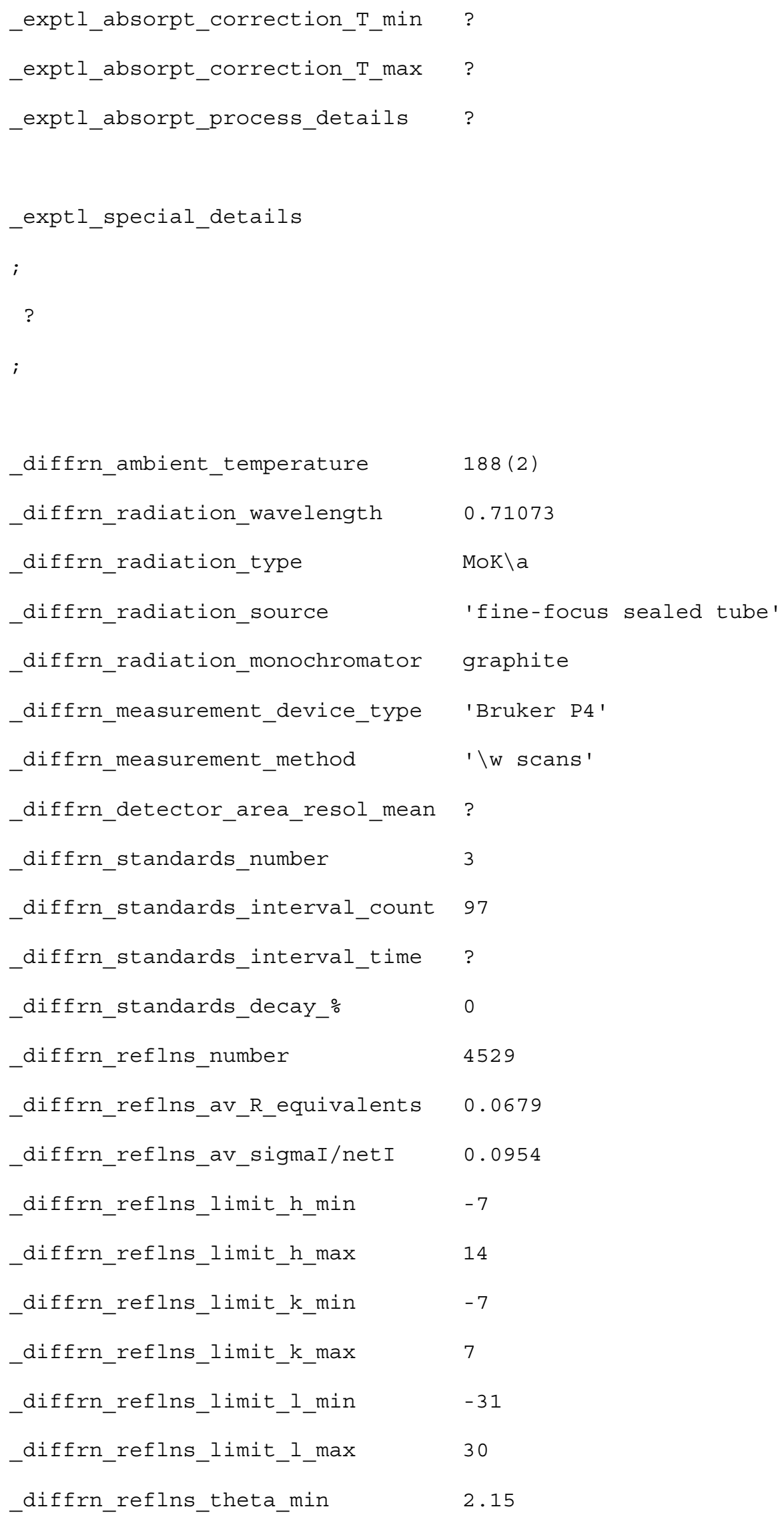




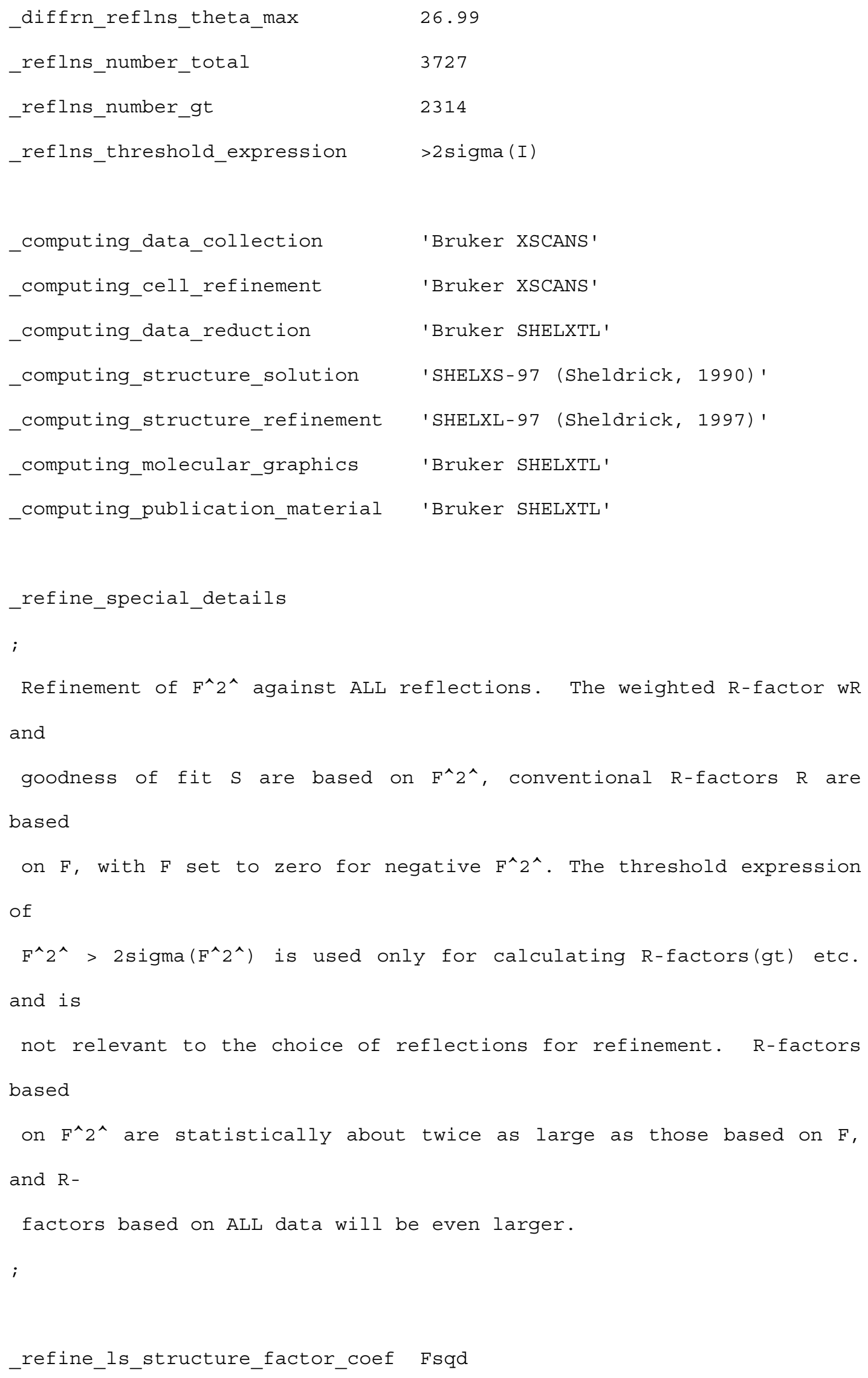




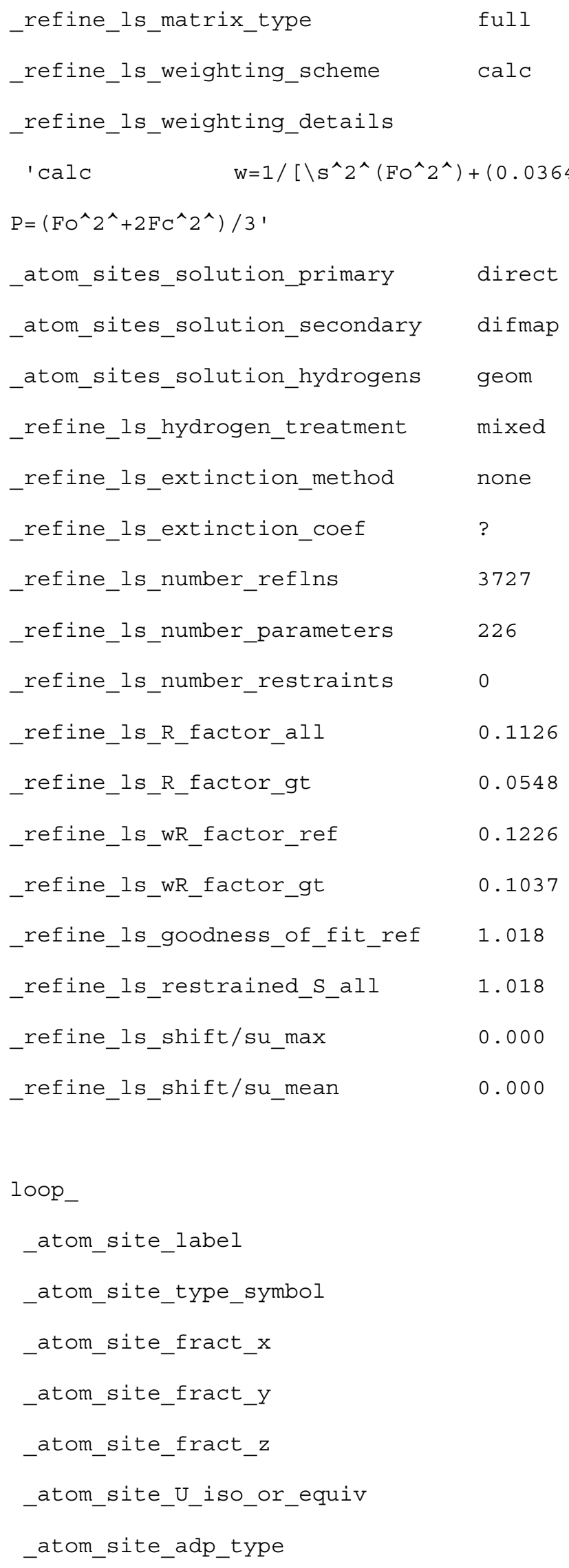




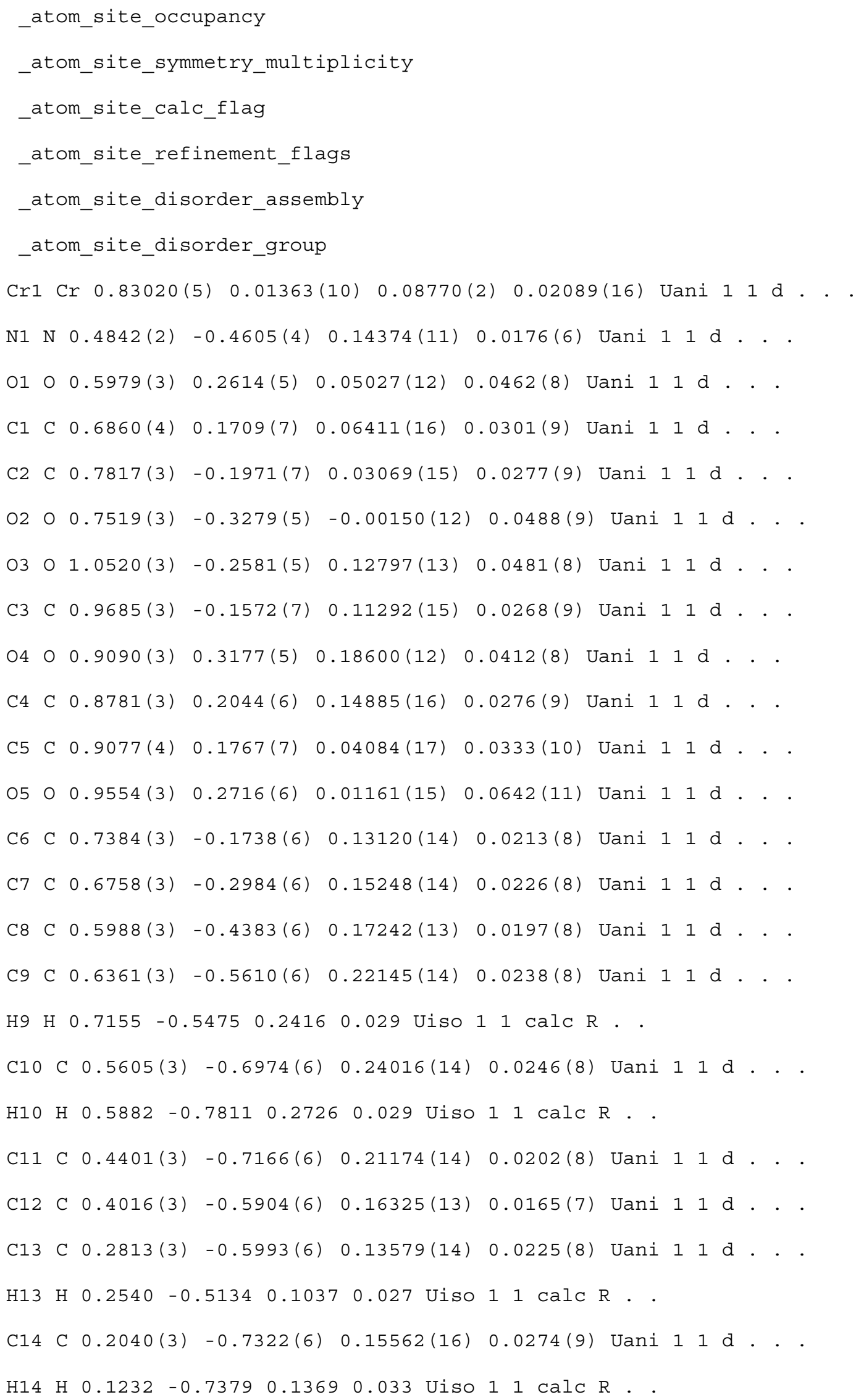




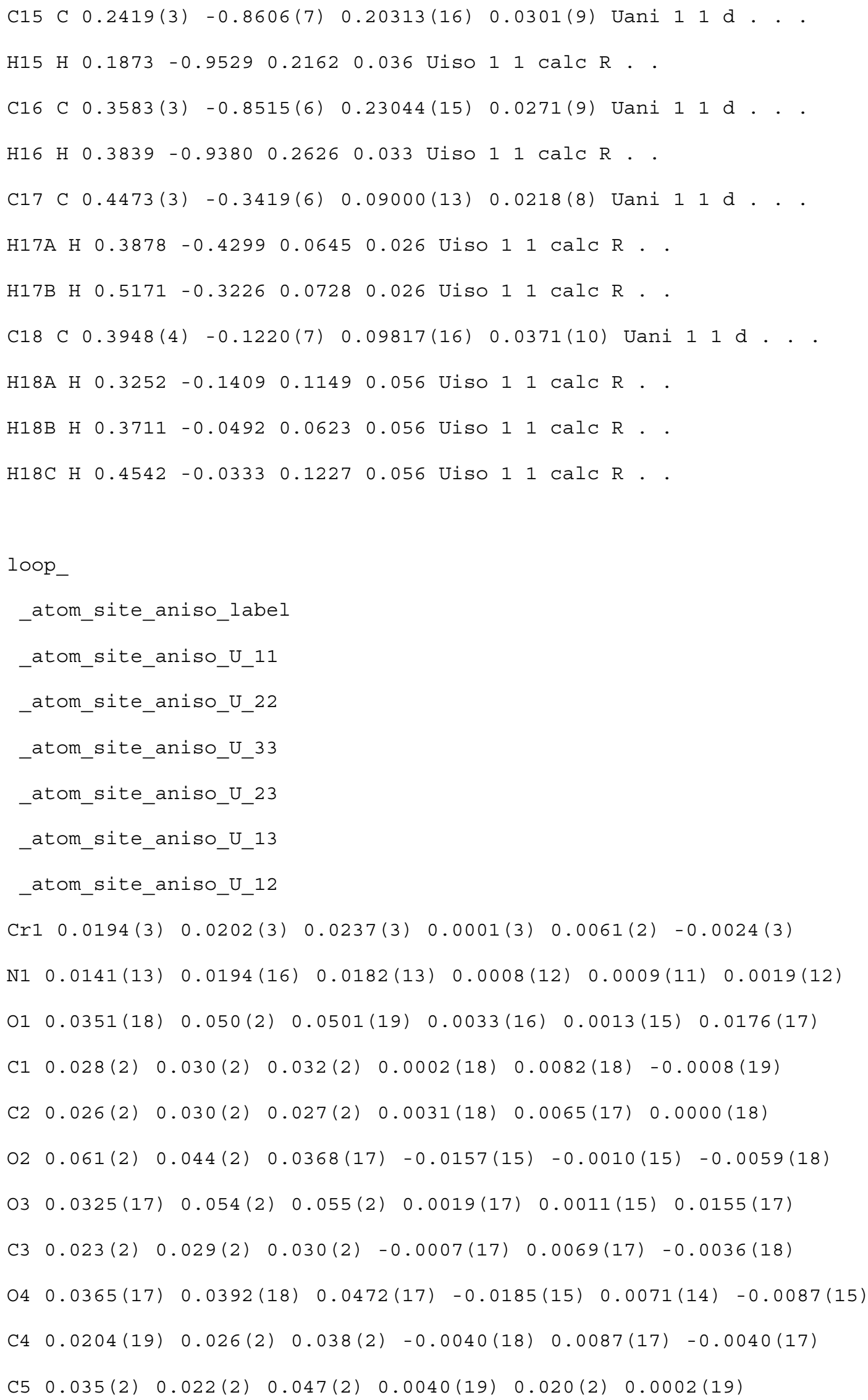




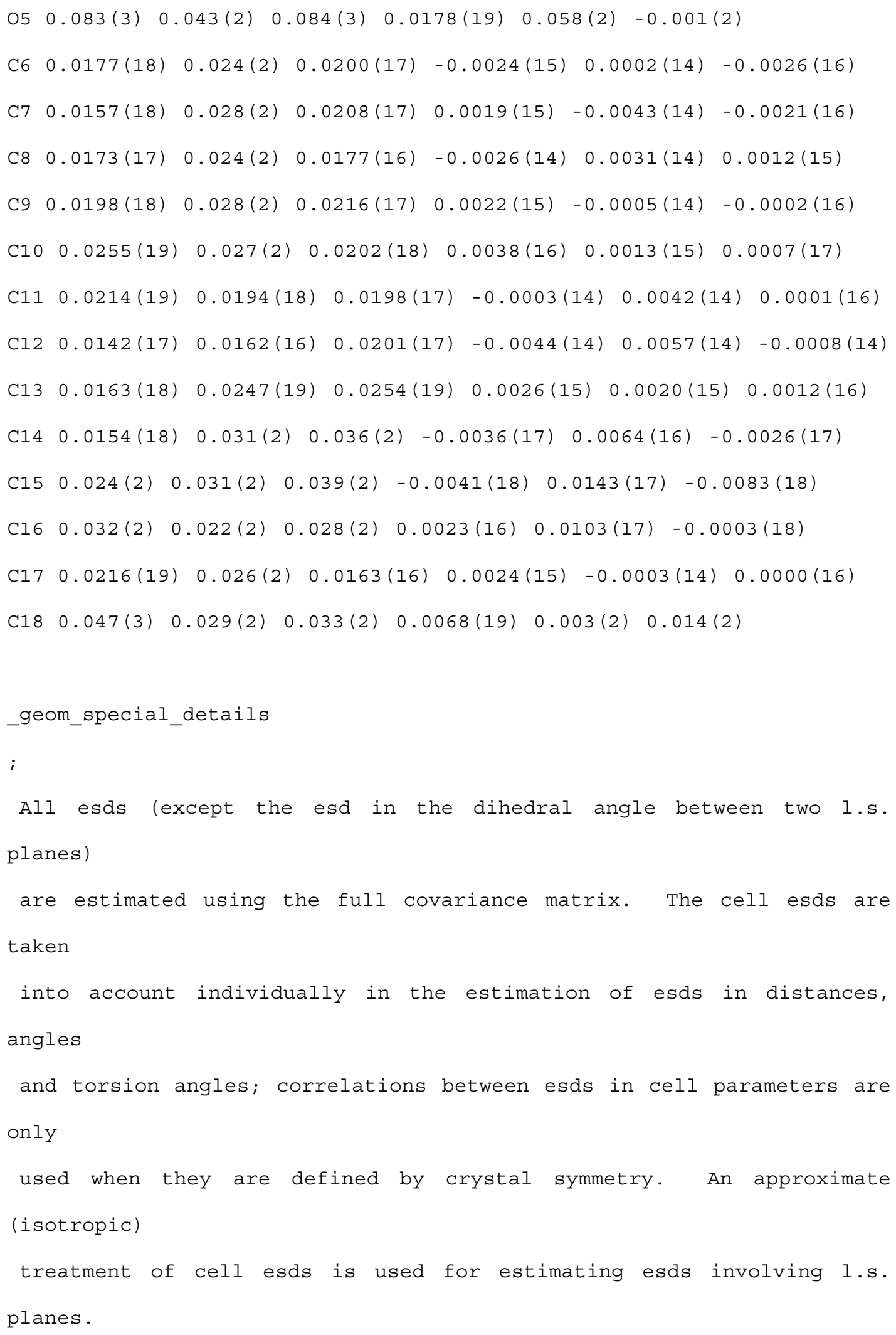




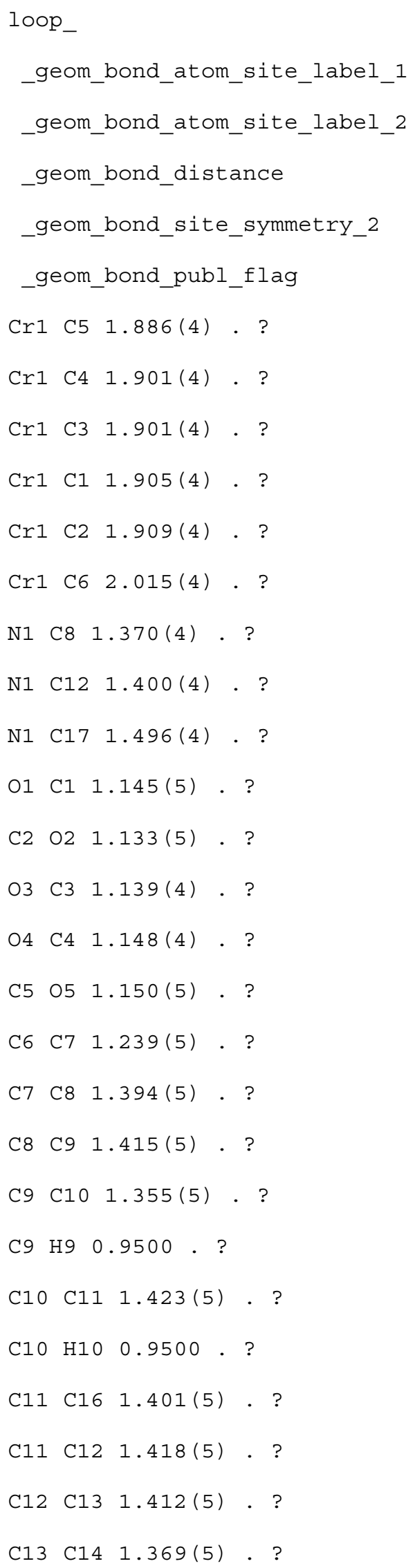




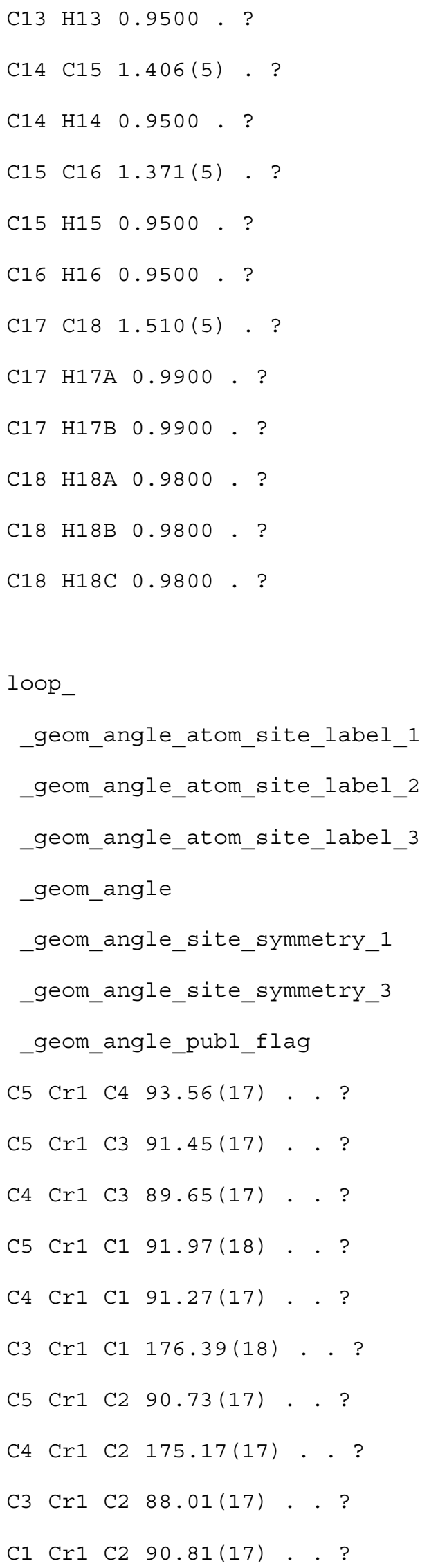




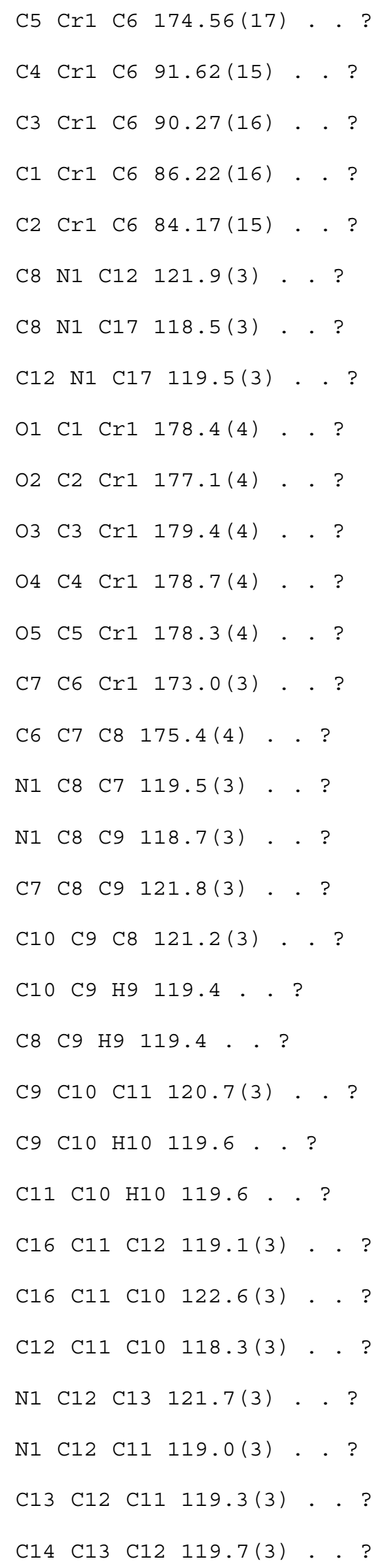




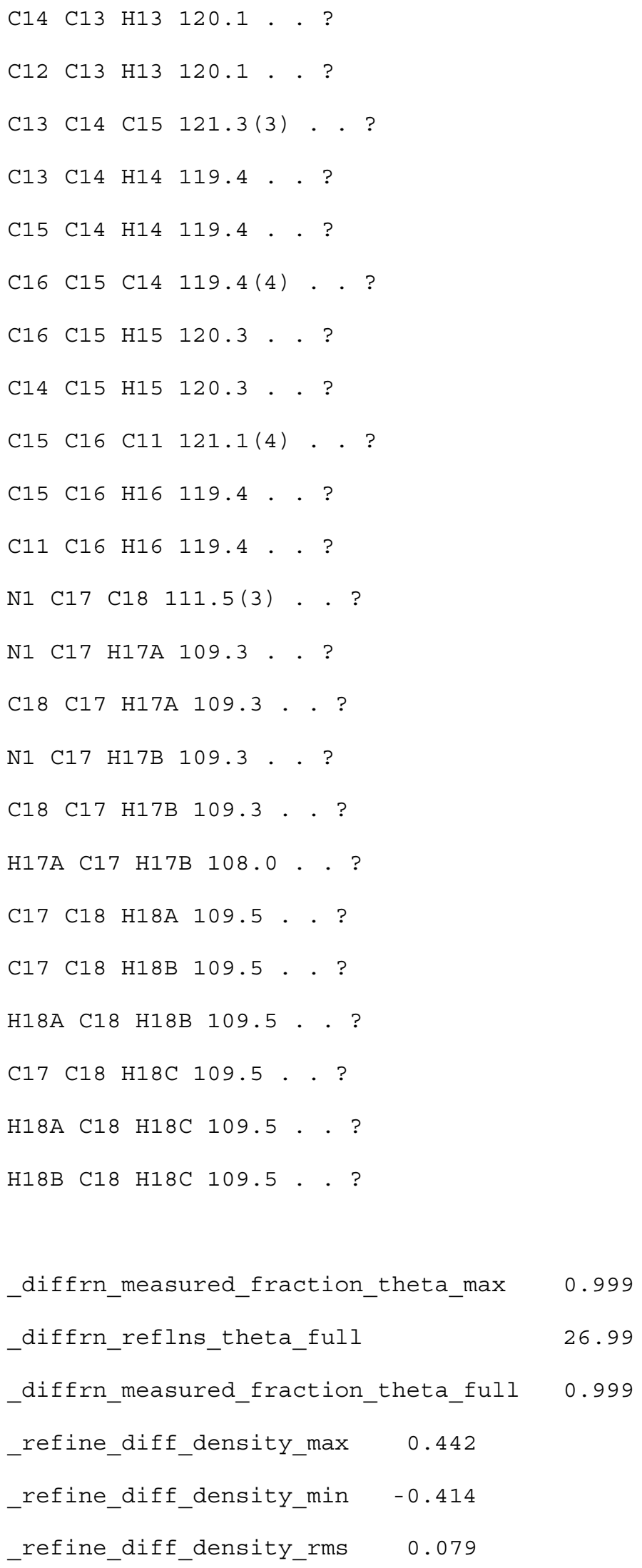




\section{Complex 19a}

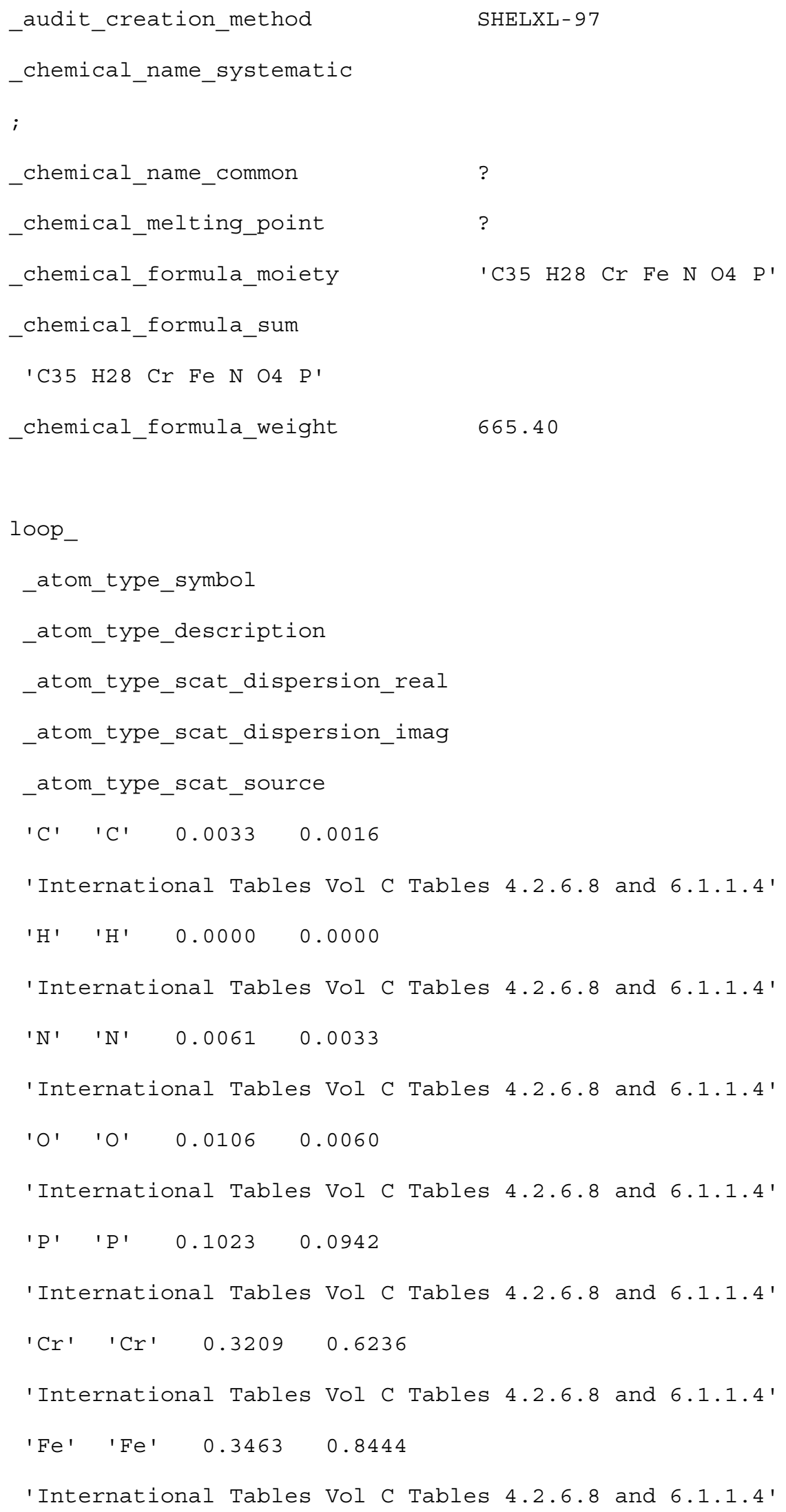




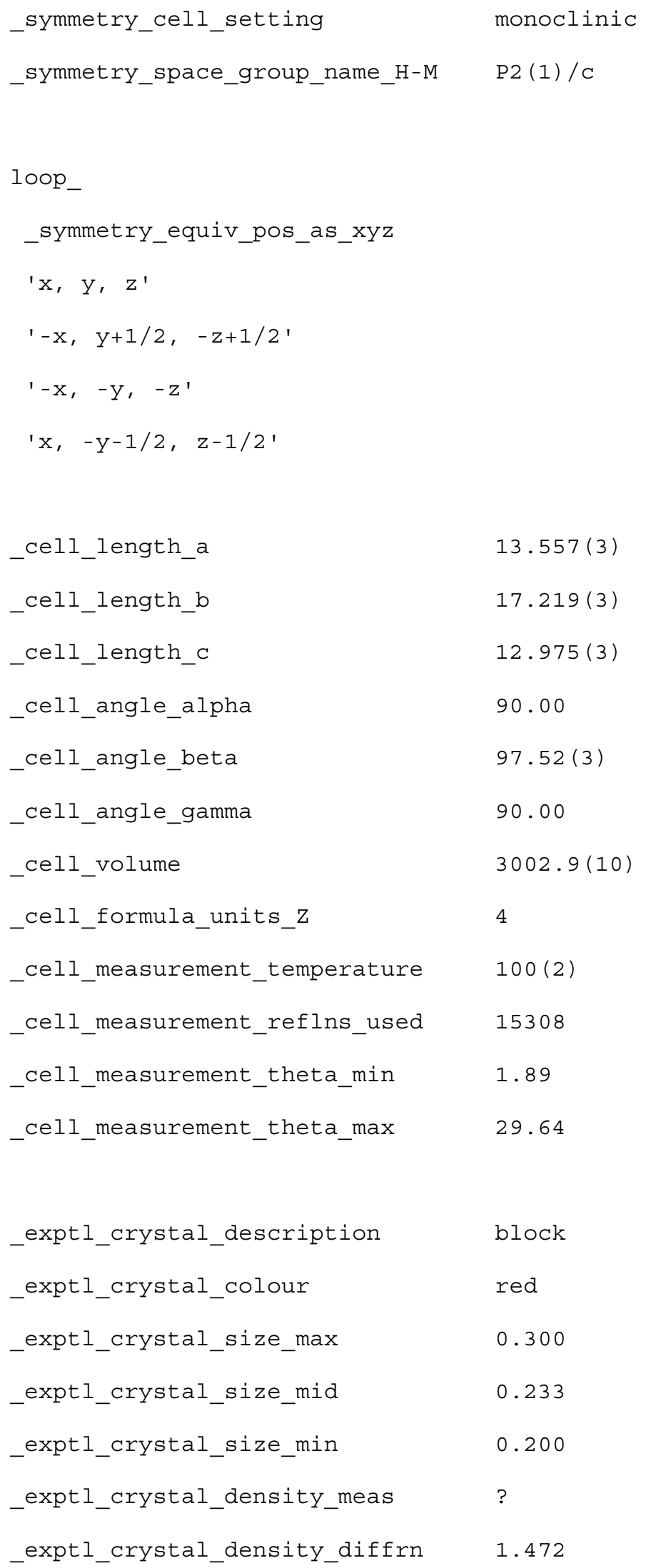




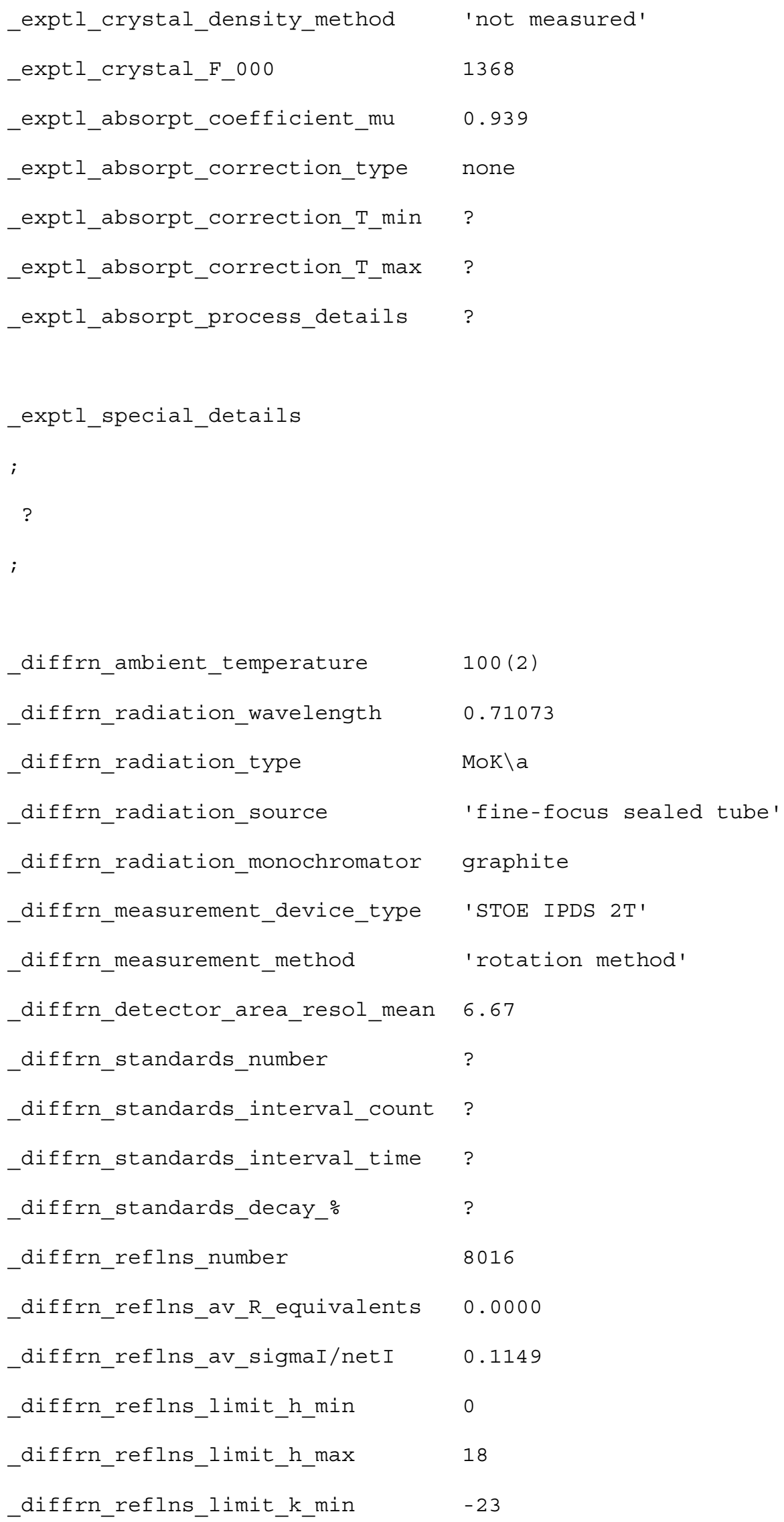




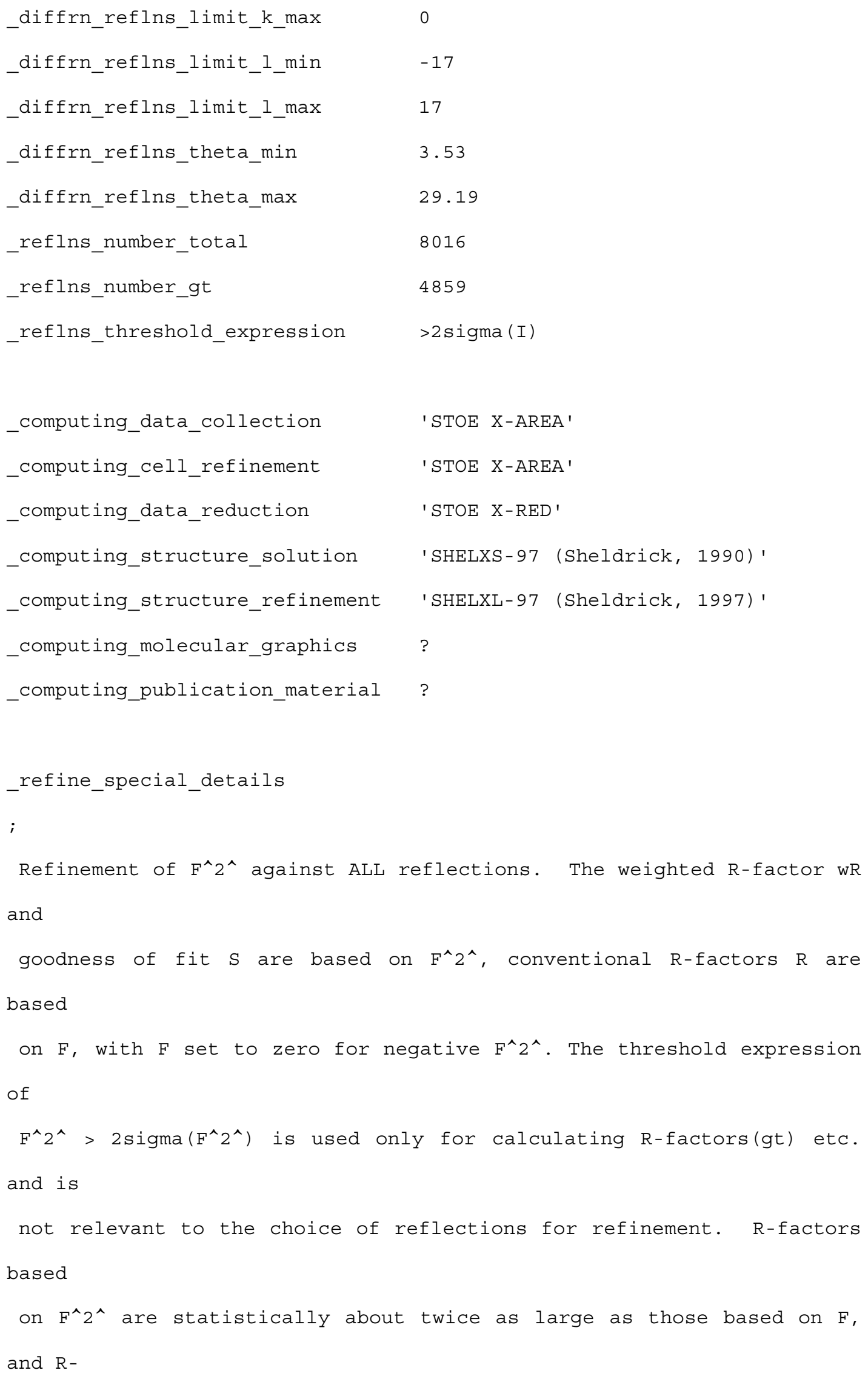




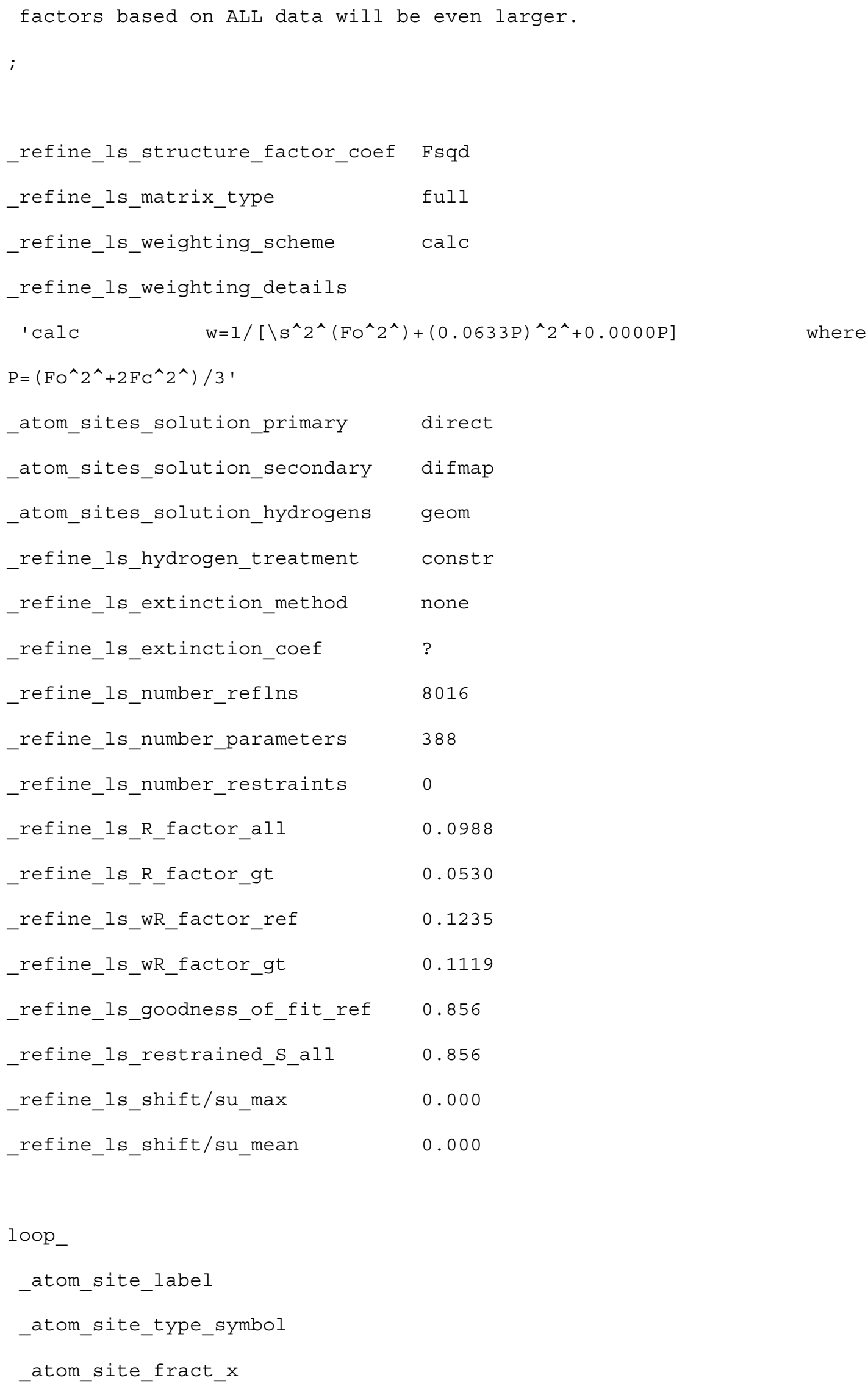




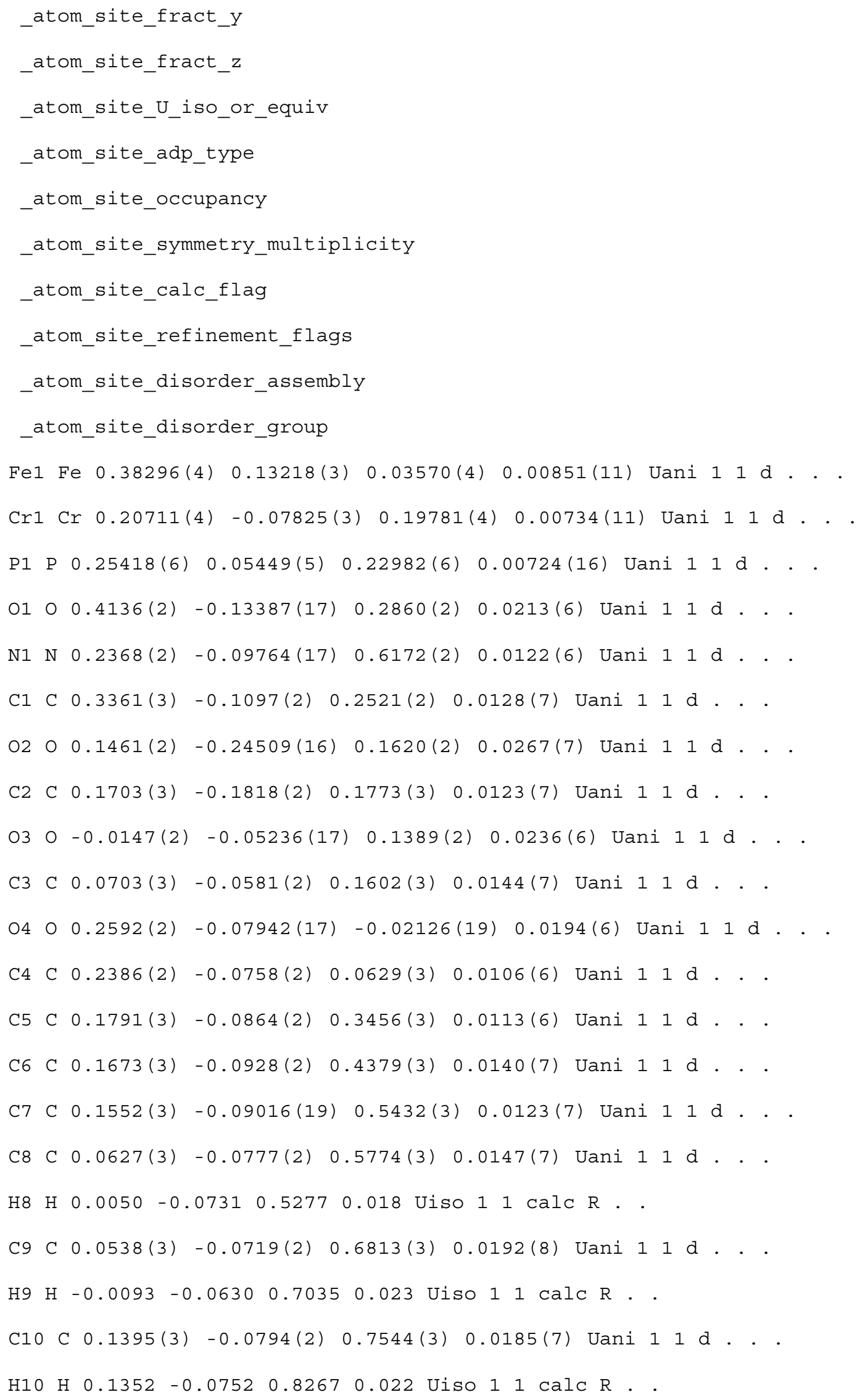


C11 C $0.2280(3) \quad-0.0926(2) \quad 0.7204(3) \quad 0.0166$ (8) Uani 11 d . . $\mathrm{H} 11 \mathrm{H} \quad 0.2857-0.0986 \quad 0.76990 .020$ Uiso 1 l calc $\mathrm{R}$. . C12 C $0.3365(3)-0.1115(2) 0.5852$ (3) 0.0203 (8) Uani 11 d. . . H12A H $0.3884-0.09310 .6406 \quad 0.024$ Uiso 11 calc $\mathrm{R}$. H12B H $0.3429-0.08150 .5214 \quad 0.024$ Uiso 11 calc R . . C13 C $0.3527(3) \quad-0.1979(3) \quad 0.5648(4) \quad 0.0305(10)$ Uani 11 d . . H13A H $0.3352-0.2286 \quad 0.62340 .046$ Uiso 11 calc $R$. H13B H $0.4227-0.20690 .55690 .046$ Uiso 11 calc $\mathrm{R}$. . H13C H $0.3106-0.21370 .5010 \quad 0.046$ Uiso 11 calc $\mathrm{R}$. . C21 C 0.1632 (2) $0.1319(2) \quad 0.1918$ (2) $0.0094(6)$ Uani 11 d. . . C22 C $0.1647(3) \quad 0.2030(2) \quad 0.2422(3) \quad 0.0159(7)$ Uani 11 d . . H22 H 0.21510 .21310 .29870 .019 Uiso 11 calc $\mathrm{R}$. . C23 C $0.0946(3) \quad 0.2595(2) \quad 0.2121(3) \quad 0.0200(8) \quad \operatorname{Uani} 11$ d . . H23 H $0.0960 \quad 0.3073 \quad 0.2490 \quad 0.024$ Uiso 11 calc R. .

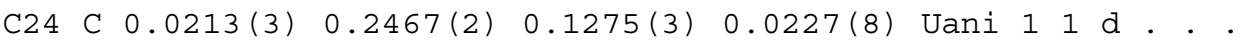
$\mathrm{H} 24 \mathrm{H}-0.02720 .28530 .10660 .027$ Uiso 11 calc $\mathrm{R}$. C25 C $0.0205(3) \quad 0.1763(2) \quad 0.0743(3) \quad 0.0194(8) \quad \operatorname{Uani} 11$ d . . H25 H $-0.02840 .16730 .0160 \quad 0.023$ Uiso 11 calc $\mathrm{R}$. C26 C $0.0903(3) \quad 0.1193(2) 0.1056(3) \quad 0.0127(7)$ Uani 11 d . . H26 H $0.0890 \quad 0.07150 .06870 .015$ Uiso 11 calc $\mathrm{R}$. . C31 C $0.2907(2) \quad 0.07479(19) \quad 0.3694(2) 0.0090(6)$ Uani 11 d. . C32 C $0.2213(3) \quad 0.0918(2) \quad 0.4354(3) \quad 0.0140(7) \quad \operatorname{Uani} 11$ d. . H32 H 0.15310 .09560 .40780 .017 Uiso 11 calc $\mathrm{R}$. . C33 C $0.2493(3) \quad 0.1034(2) \quad 0.5417(3) \quad 0.0166(7) \quad$ Uani 11 d . . H33 H $0.2007 \quad 0.1160 \quad 0.58540 .020$ Uiso 11 calc $\mathrm{R}$. . C34 C $0.3488(3) \quad 0.0964(2) \quad 0.5830(3) \quad 0.0165(7) \quad \operatorname{Uani} 11$ d. . . H34 H $0.36850 .1038 \quad 0.6554 \quad 0.020$ Uiso 11 calc R . . C35 C $0.4186(3) \quad 0.0786(2) \quad 0.5186(3) \quad 0.0142(7)$ Uani 11 d . . H35 H $0.4866 \quad 0.07390 .5468 \quad 0.017$ Uiso 11 calc $\mathrm{R}$. C36 C $0.3905(3) \quad 0.06741(19) \quad 0.4117(2) \quad 0.0107(6)$ Uani 11 d . . H36 H $0.4393 \quad 0.0548 \quad 0.36810 .013$ Uiso 11 calc $\mathrm{R}$. . 
C41 C $0.3647(2) \quad 0.08913(19) \quad 0.1790(2) \quad 0.0089(6)$ Uani $11 d .$.

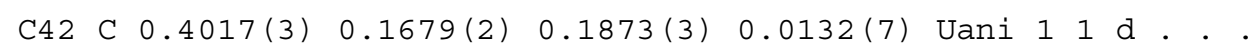
H42 H $0.36990 .2107 \quad 0.2153 \quad 0.016$ Uiso 11 calc R . . C43 C $0.4948(3) \quad 0.1705(2) \quad 0.1460(3) \quad 0.0142(7) \quad \operatorname{Uani} 11$ d. . . $\mathrm{H} 43 \mathrm{H} \quad 0.5353 \quad 0.2150 \quad 0.1413 \quad 0.017$ Uiso 11 calc R. . C44 C $0.5152(3) \quad 0.0933(2) \quad 0.1131(3) \quad 0.0139(7) \quad \operatorname{Uani} 11$ d . . $\mathrm{H} 44 \mathrm{H} 0.57240 .0780 \quad 0.0830 \quad 0.017$ Uiso 11 calc R. . C45 C $0.4361(3) \quad 0.0435(2) \quad 0.1328(3) \quad 0.0116(7)$ Uani 11 d. . . H45 H $0.4313-0.01050 .11800 .014$ Uiso 11 calc $\mathrm{R}$. C51 C $0.3445(3) \quad 0.0925(2) \quad-0.1146(3) \quad 0.0184(8)$ Uani 11 d. . . H51 H $0.3590 \quad 0.0430 \quad-0.14120 .022$ Uiso 11 calc $\mathrm{R}$. C52 C $0.2574(3) \quad 0.1124(2) \quad-0.0690(3) \quad 0.0150(7)$ Uani 11 d . . H52 H $0.2040 \quad 0.0787-0.05910 .018$ Uiso 11 calc R . . C53 C $0.2659(3) \quad 0.1923(2) \quad-0.0411(3) \quad 0.0159(7)$ Uani 11 d. . . H53 H $0.21820 .2213-0.00970 .019$ Uiso 11 calc $\mathrm{R}$. C54 C $0.3567(3) \quad 0.2216(2) \quad-0.0674(3) \quad 0.0169(7)$ Uani 11 d. . . H54 H $0.3806 \quad 0.2733-0.0566 \quad 0.020$ Uiso 11 calc $\mathrm{R}$. C55 C $0.4060(3) \quad 0.1601(2)-0.1131(3) \quad 0.0152(7)$ Uani 11 d. . . H55 H $0.46860 .1633-0.13810 .018$ Uiso 11 calc $\mathrm{R}$. .

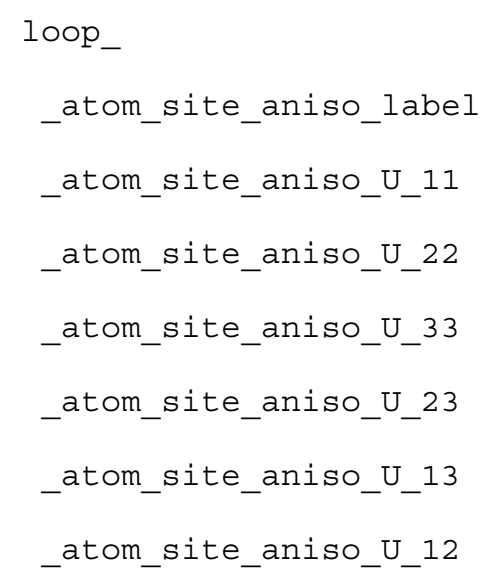




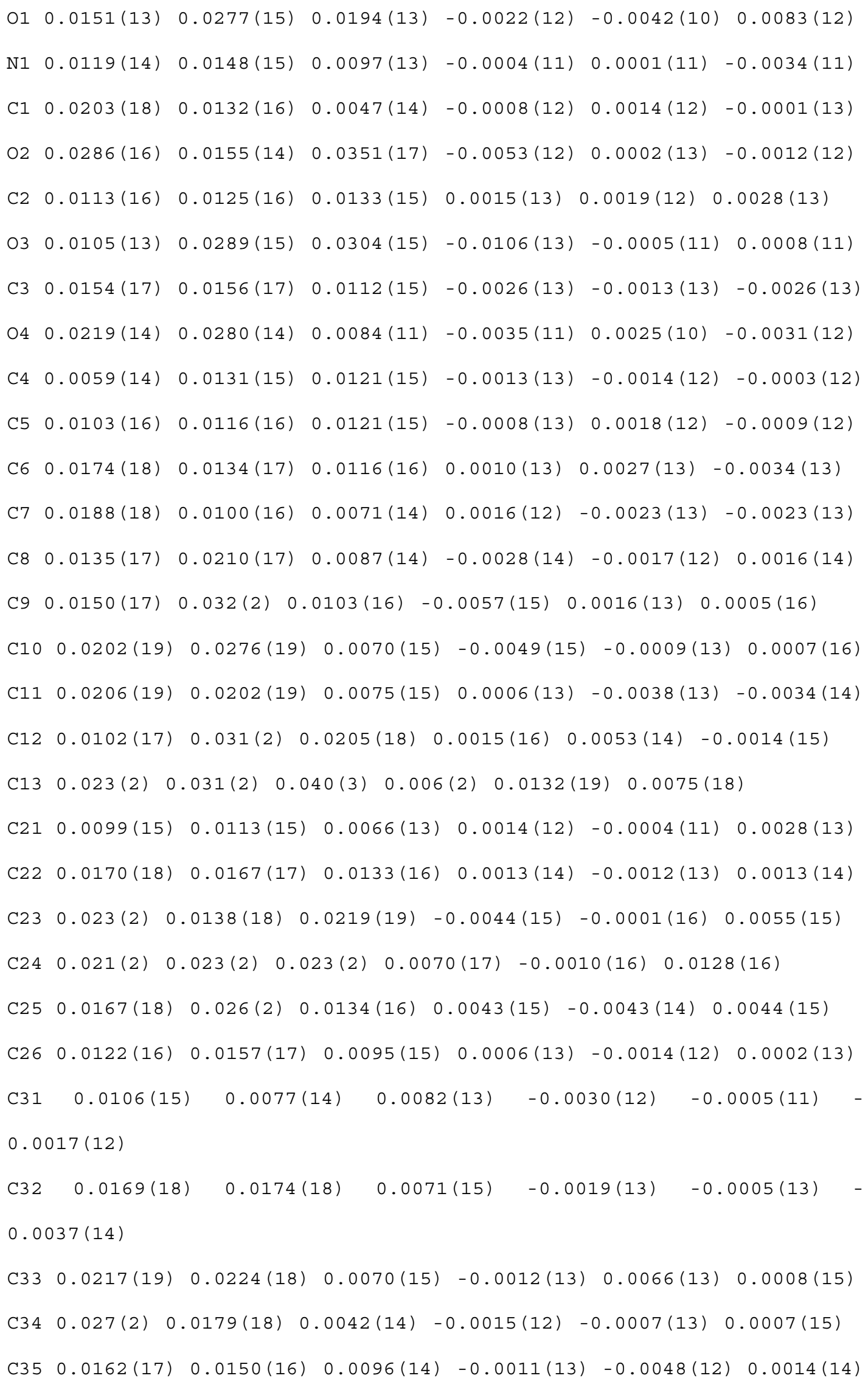




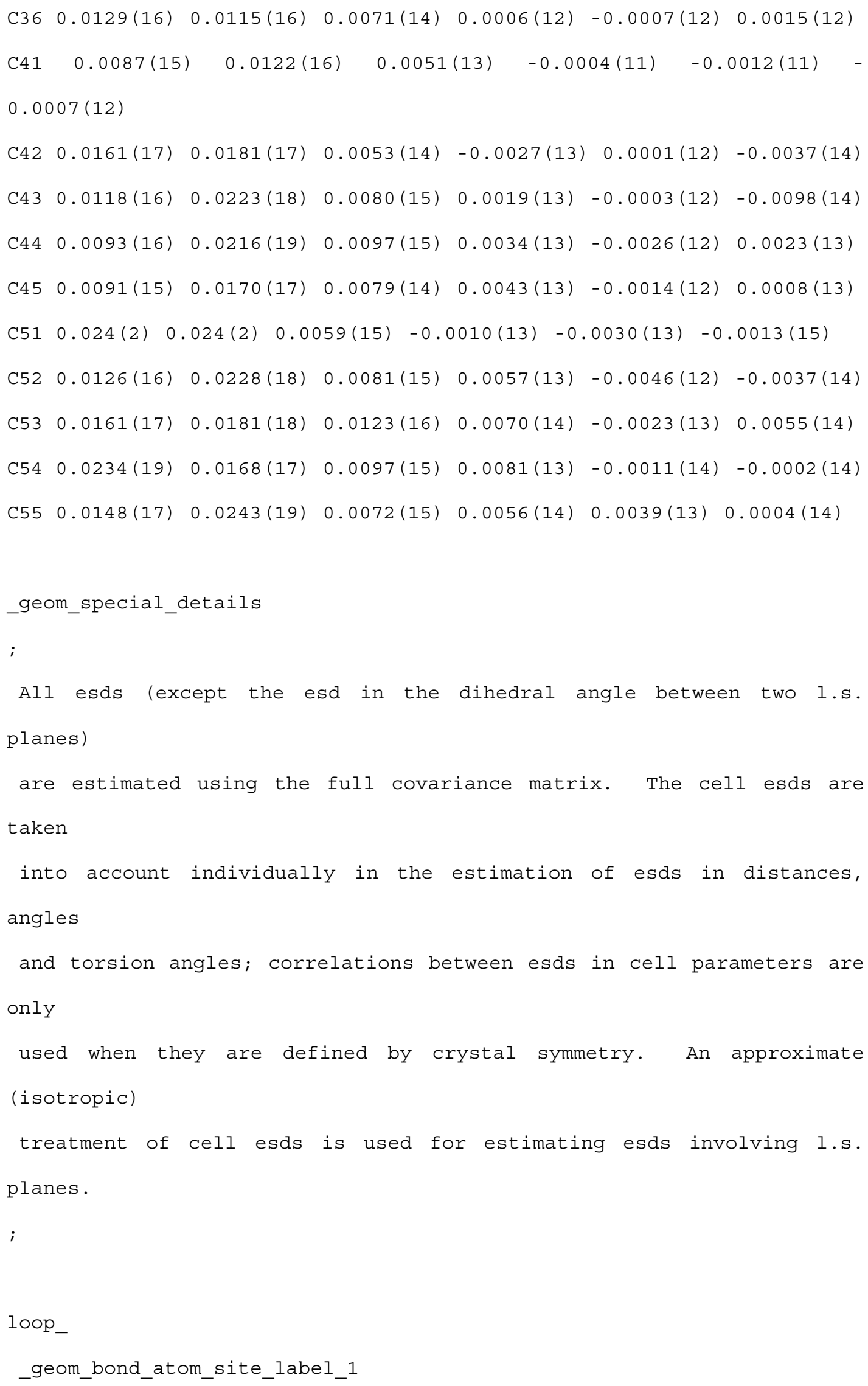




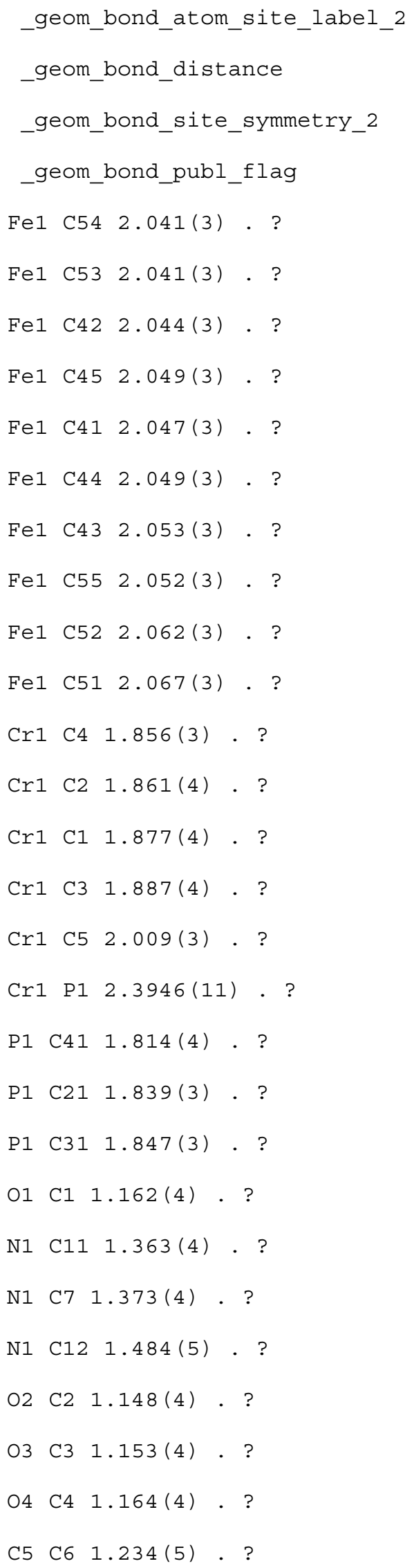




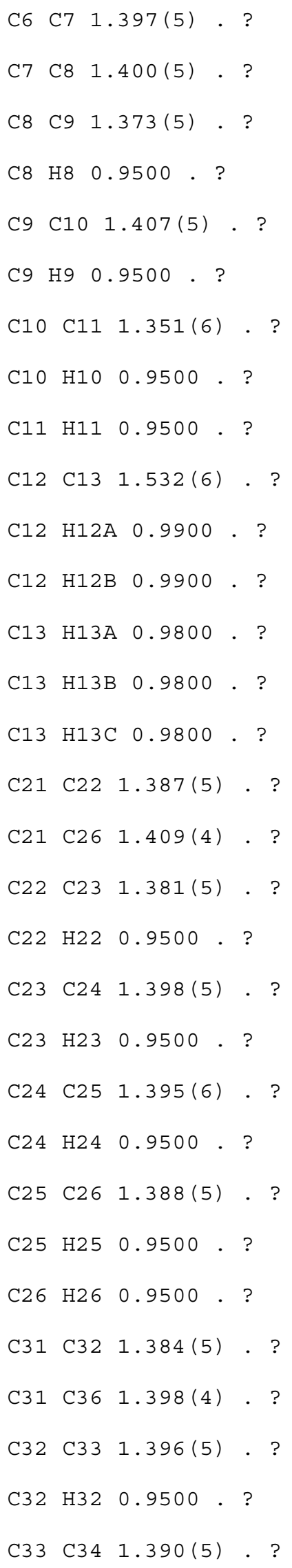




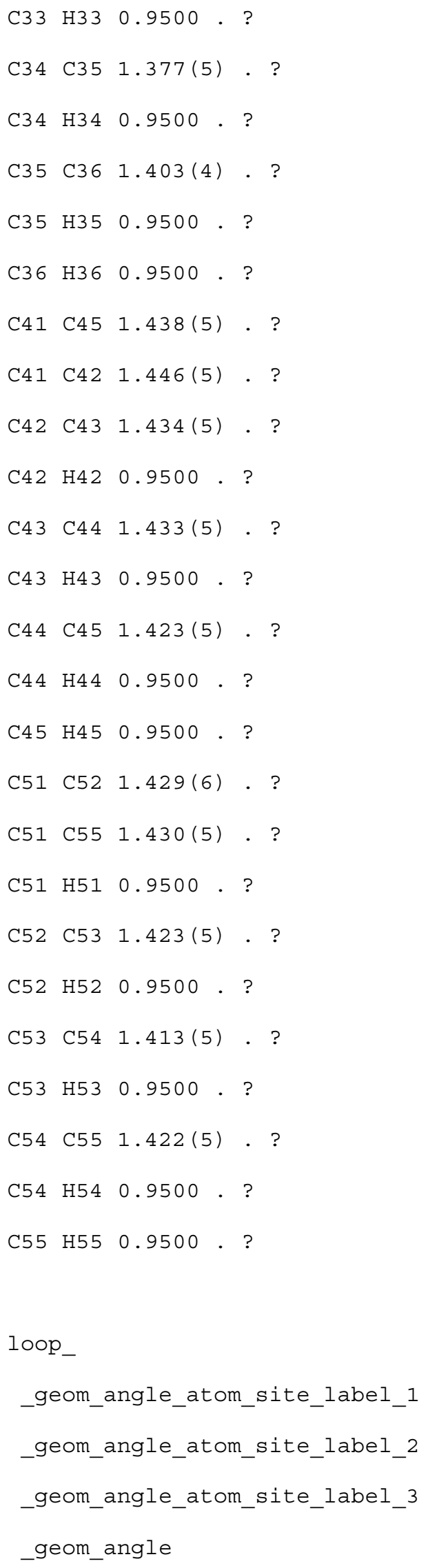




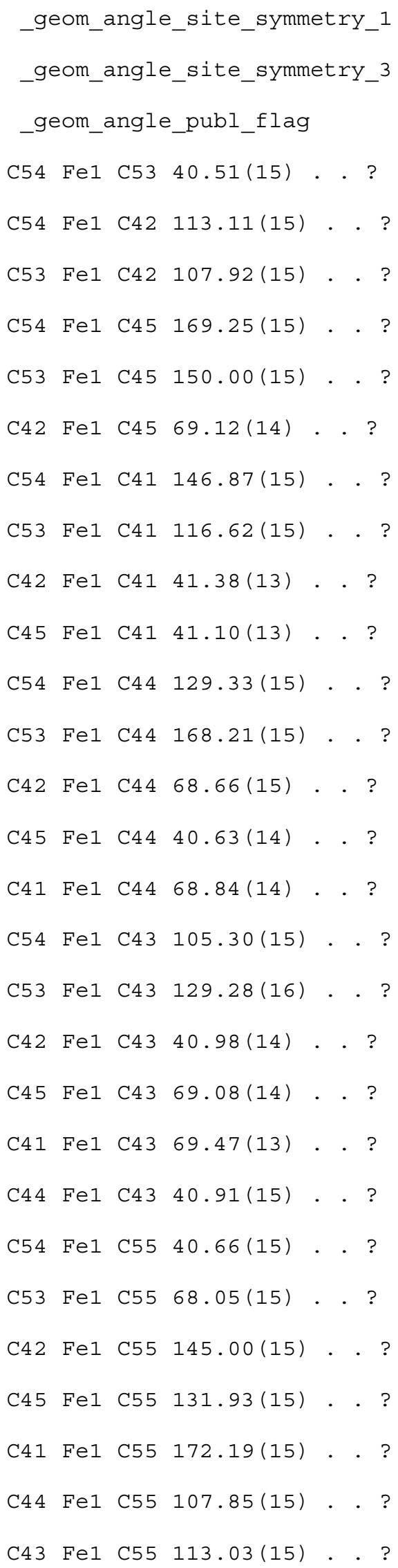




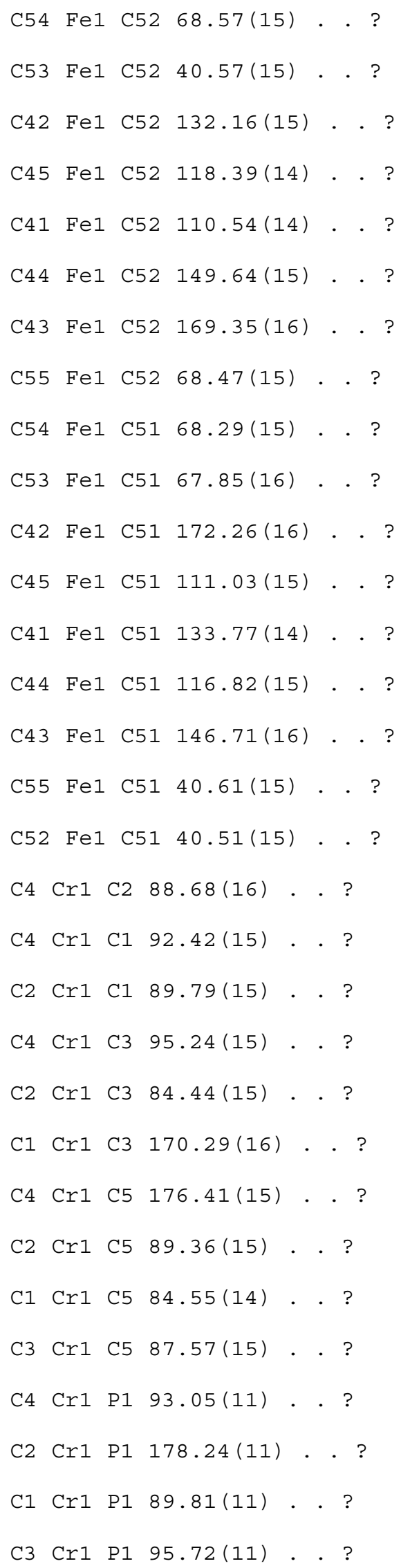




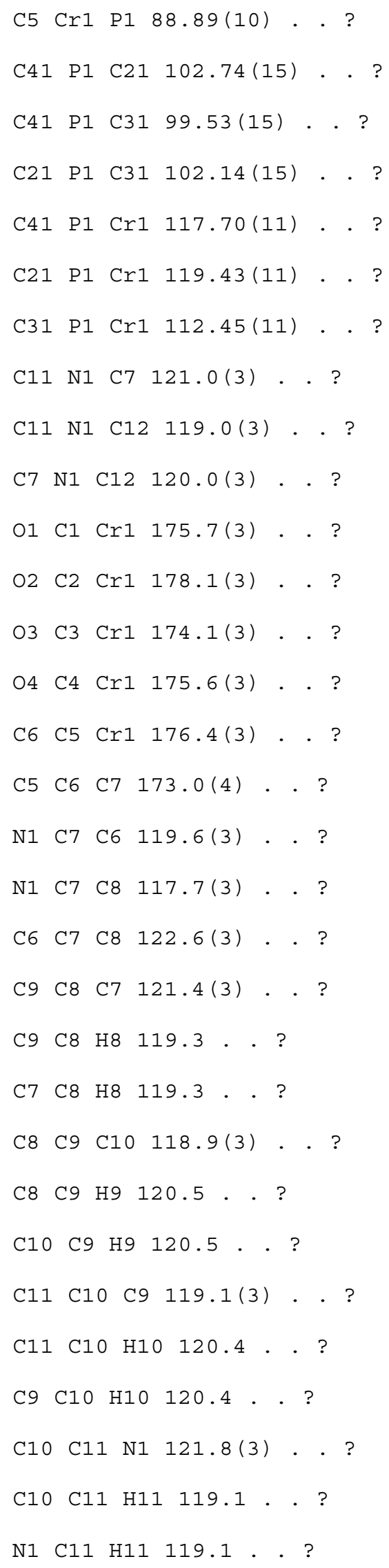




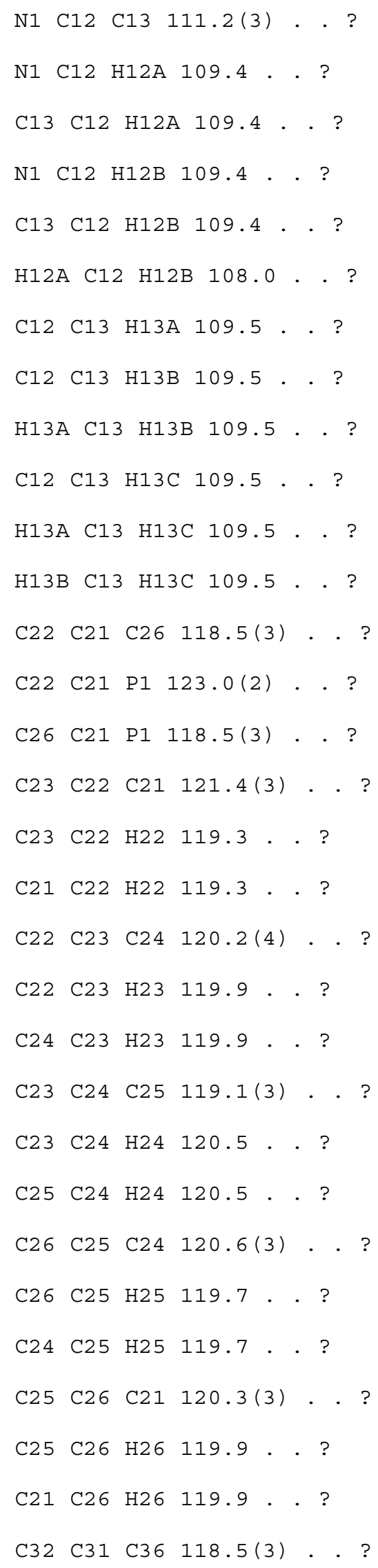




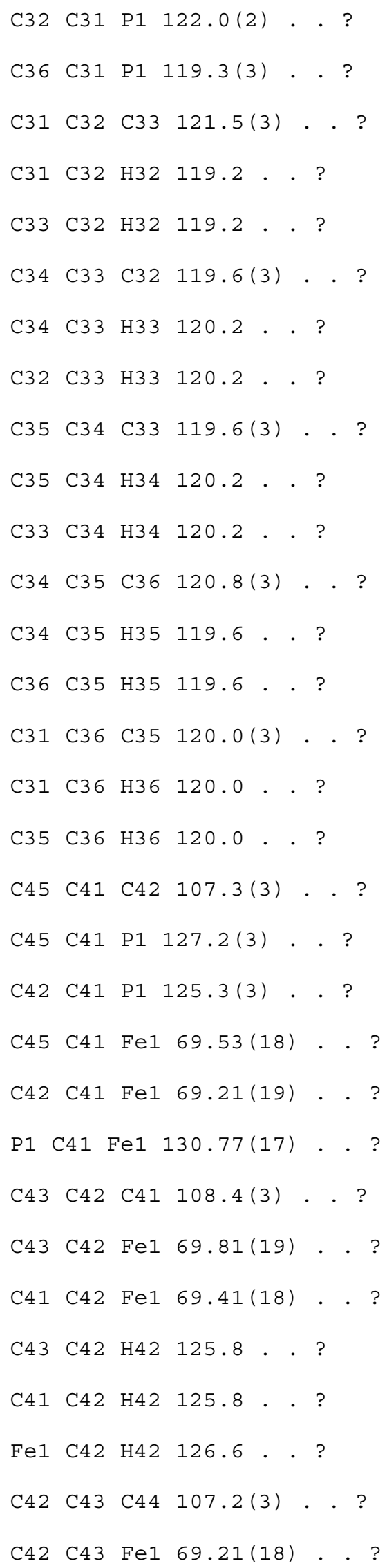




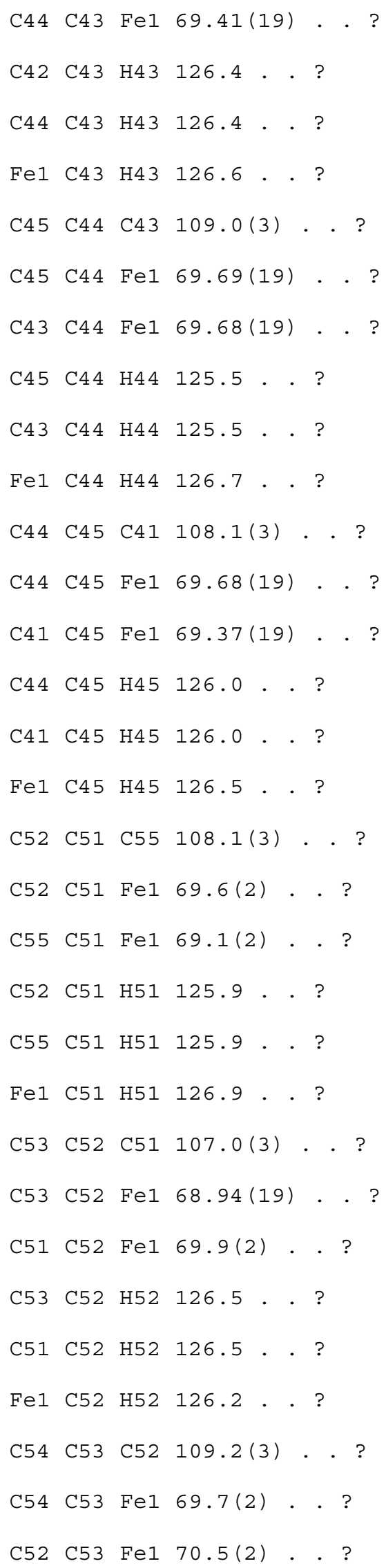




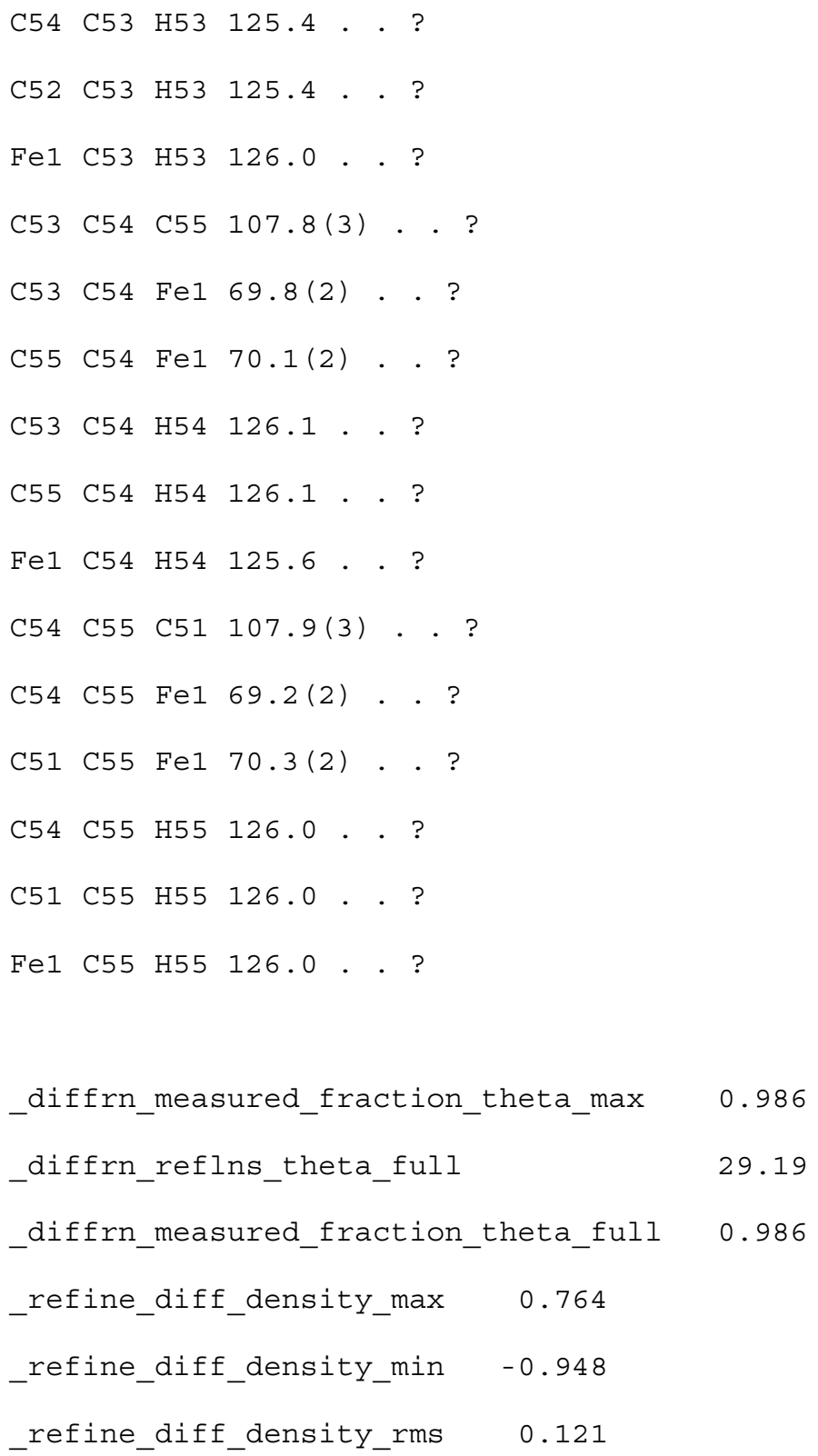

